Check for updates

Cite this: RSC Adv., 2019, 9, 38447

\title{
Simple preparation of graphene quantum dots with controllable surface states from graphite $\uparrow$
}

\author{
Sukhyun Kang, $t^{a}$ Young Kyu Jeong, $\ddagger^{a}$ Kyung Hwan Jung, ${ }^{a}$ Yong Son, ${ }^{b}$ \\ Sung-Churl Choi, ${ }^{c}$ Gae Seok An, ${ }^{C}$ Hyuksu Han (D) ${ }^{* d}$ and Kang Min Kim (D) *a
}

Graphite is economic and earth-abundant carbon precursor for preparing graphene quantum dots (GQDs). Here, we report a facile and green approach to produce GQDs from graphite flakes via a pulsed laser ablation (PLA) method assisted by high-power sonication. A homogeneous dispersion of graphite flakes, caused by high-power sonication during PLA, leads to the formation of GQDs following a laser fragmentation in liquid (LFL) rather than laser ablation in liquid (LAL) mechanism. The final product of GQDs exhibits the distinct structural, chemical, and optical properties of pristine graphene itself. However, graphene oxide quantum dots (GOQDs) with abundant surface oxygen-rich functional groups are readily formed from graphite flakes when high-power sonication is not employed during the PLA process. GQDs and GOQDs show a significantly different luminescence nature. Hence, selective production of either functional GQDs or GOQDs can be achieved by simply turning the high-power sonication during the PLA process on and off. We believe that our modified PLA process proposed in this work will further open up facile and simple routes for designing functional carbon materials.

Received 18th September 2019 Accepted 18th November 2019

DOI: $10.1039 / \mathrm{c} 9 \mathrm{ra07555k}$

rsc.li/rsc-advances these methods usually suffer from the use of expensive carbonprecursors such as graphene oxide (GO), reduced graphene oxide (rGO) or highly oriented pyrolytic graphite (HOPG), increasing cost of the final products of GQDs. Hence, it is urgently necessary to develop facile, green, and economic synthetic method for the synthesis of GQDs, but remains a challenge.

Pulsed laser ablation (PLA) method, which is a simple and clean process, has been recently proven as a versatile and effective technique to synthesize GQDs. ${ }^{18,19}$ In addition, graphite is earth-abundant and inexpensive carbon-precursor for preparing GQDs. ${ }^{20}$ Functional GQDs have been prepared from graphite using typical wet-chemical methods. ${ }^{16}$ However, only a few papers have been reported for the preparation of GQDs using graphite via PLA methods. Recently, V. Thongpool and co-workers synthesized GQDs from the graphite target by PLA methods. ${ }^{21} \mathrm{~T}$ method requires time consuming experimental steps to prepare graphite target by hot isostatic press and post-heat treatment, diminishing economical merits of their method.

Here, we first report ultimately cost-effective and green approach to produce GQDs via PLA process combined with high-power sonication using pristine graphite flakes as starting materials. To our best knowledge, the synthesis of GQDs using unrefined pristine graphite flakes (i.e., not a solid sintered target) through the PLA has never been reported. We believe that our method is the most economic and simple way to synthesize GQDs among the recently reported methods.

\footnotetext{
${ }^{a}$ Korea Institute of Industrial Technology, Gwahakdanji-ro 137-41, Gangwon-do, 25440, Republic of Korea. E-mail: kmkim@kitech.re.kr

do, 15014, Republic of Korea

${ }^{c}$ Division of Materials Science and Engineering, Hanyang University, 17 Haengdangdong, Seongdong-gu, Seoul 133-791, Republic of Korea

${ }^{d}$ Department of Materials Science and Engineering, Hongik University, Sejong-ro 2636, Sejong, Republic of Korea. E-mail: hhan@hongik.ac.kr

$\dagger$ Electronic supplementary information (ESI) available. See DOI: 10.1039/c9ra07555k

$\$$ S. Kang and Y. K. Jeong contributed equally to this work.
} 
Briefly, $500 \mathrm{mg}$ of graphite flakes was dispersed in $200 \mathrm{ml}$ of ethanol by high-power tip-type ultra-sonication during the laser ablation process ensuring for homogeneous dispersion of the graphite flakes in solvent. Suspension of the graphite flakes in a vertical column was ablated by using horizontal pulsed laser with a wavelength of $355 \mathrm{~nm}$. The pulsed laser beam was focused on the center of graphite suspension.

\section{Results and discussion}

Fig. 1 shows high resolution transmission electron microscopy (HR-TEM) images of GQDs prepared by PLA for $30 \mathrm{~min}$ with or without high-power sonication. The produced GQDs in both sonication on (on-GQDs, Fig. 1a) and sonication off (off-GOQDs, Fig. 1b) have average diameters below $10 \mathrm{~nm}$ with homogeneous size and shape. The size distributions of both GQDs are shown in Fig. S1.† An average diameters of $3.8 \pm 0.4$ and $4.1 \pm$ 0.3 are obtained for on-GQDs and off-GOQDs, respectively, by counting more than 55 of GQDs. Also, according to the fastFourier transformation (FFT) patterns (the right insets of Fig. 1c and d), both of on-GQDs and off-GOQDs are crystallized in the pristine graphene structure with a lattice parameter of $\sim 0.24 \mathrm{~nm}$ as confirmed by the HR-TEM images (the left insets of Fig. 1c and d). Noted that no crystalline features that correspond to graphite, such as [002] plane, were observed for both of on-GQDs and off-GOQDs by HR-TEM. ${ }^{22}$ Atomic force microscopy (AFM) was performed to illustrate the topographic morphology and height distributions of on-GQDs and off-GQDs (Fig. S2 $\dagger$ ). The height line profile reveals that the thickness of on-GQDs and off-GOQDs are less than $2 \mathrm{~nm}$ corresponding to 45 graphene layers. Statistical analysis of AFM data indicates that more than $80 \%$ of the on-GQDs and off-GOQDs has a thickness between 0.5 and $2.0 \mathrm{~nm}$. Yield of our PLA process was also calculated by dividing the weight of dried on-GQDs and offGQDs products by the weight of the starting MWCNTs. Based on this method, the yield of on-GQDs and off-GOQDs were determined as about $8-12 \%$.

X-ray photoelectron spectroscopy (XPS) was employed for studying chemical bonding nature of on-GQDs and off-GOQDs. XPS spectra show that both on-GQDs and off-GOQDs present three main peaks associated with carbon atomss, which are located at 284.1, 286.0 and $288 \mathrm{eV}$, corresponding to $\mathrm{sp}^{2}$ carbon, hydroxyl and carboxyl functional groups bonded in $\mathrm{sp}^{3}$ carbon structure, respectively (Fig. 2a and b). Table $51 \dagger$ depicts the quantitative analysis of the XPS spectra, where fractions of carboxyl (\%) and hydroxyl (\%) groups in on-GQDs and offGOQDs are summarized. Interestingly, total fractions of carboxyl and hydroxyl groups were increased from $12.6 \%$ (onGQDs) to $46.73 \%$ (off-GOQDs). Raman spectroscopy was also conducted to further investigate the structural defect of onGQDs and off-GOQDs as shown in Fig. S6. $\dagger$ The on-GQDs show a disorder (D) band at $1349 \mathrm{~cm}^{-1}$ and a $\mathrm{sp}^{2}$ bond (G) band at $1576 \mathrm{~cm}^{-1}$ as well as $I_{\mathrm{D}} / I_{\mathrm{G}}$ ratio of 0.72 , which smaller than that of the off-GOQDs (0.9). These results imply that the synthesized on-GQDs have a pure $\mathrm{sp}^{2}$ carbon crystalline structure with fewer oxygen defects. Also, the broadening of the D peak (off-GOQDs) is due to an increase in carbon to oxygen groups such as carboxyl and hydroxyl functional groups. ${ }^{35}$ This reveals that during PLA process without tip-type sonication, oxygen-rich functional groups are introduced on the surface of GQDs. In other words, chemical compositions and structure of the GQDs can be precisely tuned by applying high-power (a)

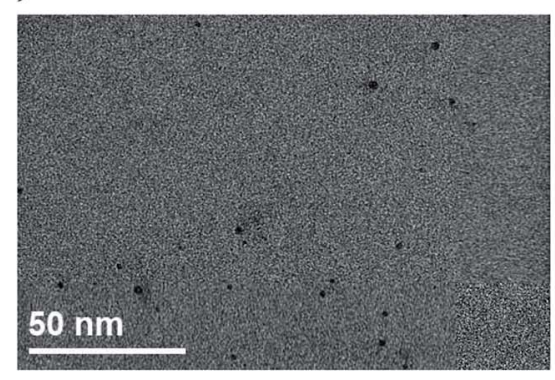

(c)

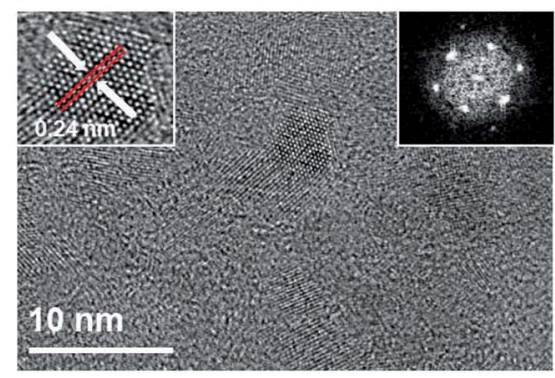

(b)

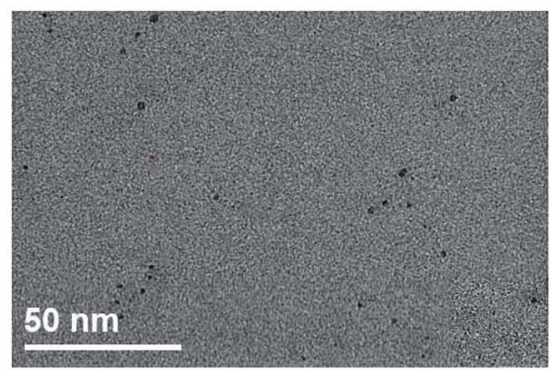

(d)

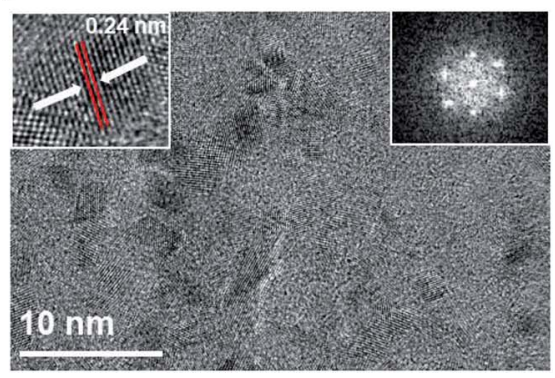

Fig. 1 HR-TEM images of on-GQDs and off-GOQDs. (a) TEM images of on-GQDs and (b) off-GOQDs. They both showing the uniform morphology shape and size. (c) HR-TEM image of on-GQDs and (d) off-GOQDs. Insets are the 2D FFT pattern (right). They both show high quality crystalline hexagonal patterns of graphene quantum dots. Left side insets show the lattice distance of on-GQDs and off-GOQDs. 
(a)

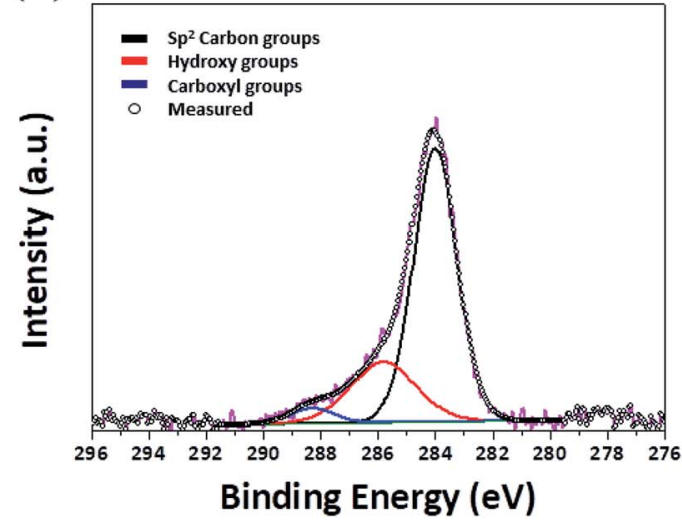

(b)

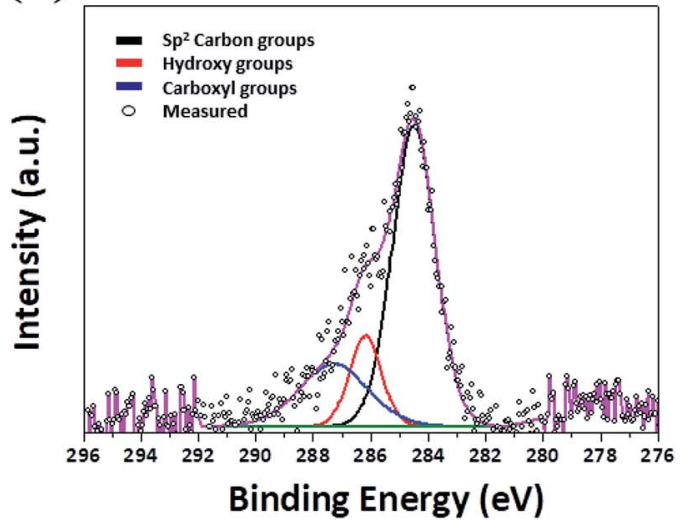

Fig. 2 XPS spectra (a) on-GQDs and (b) off-GOQDs.

sonication for graphite flakes during PLA process. Therefore, it is necessary to investigate transformation mechanism of graphite flakes to GQDs under vigorous dispersion condition during PLA process.

Possible mechanisms can be explained by laser fragmentation in liquid (LFL) and laser ablation in liquid (LAL) process, respectively. In top-down processes, the LFL process has been generally adopted to explain for physicochemical phenomena of size-reduction and size-distribution of colloidal nanoparticles by the nano- or pico-second laser. ${ }^{23,24}$ It should be noted here that facile convection of precursors is essential to obtain homogenous colloidal nanoparticles by LFL. In contrast, LAL process is generally used for explaining the formation of colloidal nanoparticles in bottom-up processes, which is associated with the generation of cavitation bubble and plasma plume near the surface of target due to sequential process of thermal heating, melting and evaporation by laser irradiation. ${ }^{25,26}$ Notably, the vigorous convection of graphite flakes driven by the tip-type sonication can remarkably reduce exposed time of graphite flakes to laser irradiation. This can also effectively suppress thermal heating of the graphite flakes during laser irradiation. Consequently LFL process rather than LAL process dominates the formation mechanism of on-GQDs when high-power sonication is employed for PLA (Fig. 3a). On the other hand, graphite flakes may prefer to be aggregated each other at the bottom of glass vial when the high-power sonication is off. In this condition, cavitation bubble and plasma plume are easily generated on the surface of the graphite aggregates during laser irradiation, where LAL process is likely to be the dominant formation mechanism of off-GOQDs (Fig. 3b).

Hence, we highly assumed that the intermediates of graphite flakes synthesized by PLA with or without high-power sonication shows substantially different morphologies. Morphology changes of graphite flakes were monitored by ex situ TEM analysis, which are prepared by laser irradiation for $5 \mathrm{~min}$ and 30 min. For LFL process, homogeneous transitions from graphite flake to on-GQDs were clearly observed as shown in Fig. 3c-e. At the initial state, the laser is injected to homogeneously distributed graphite flakes, where a high pressure can be applied on the surface of individual graphite flake. ${ }^{27}$ Large graphite flakes are readily exfoliated into well-dispersed small graphene sheets by a high energetic collision (Fig. 3d). In sharp contrast for off-GOQDs, the laser energy can be slowly, relative to LFL process, transferred to the surface of graphite flakes, leading to the formation of carbon clusters in cavitation bubble. These carbon clusters with high surface energy tend to aggregate each other, resulting in the highly aggregated graphene nanosheets when the temperature decreases and the internal pressure of the bubble drops to the value lower than those of the surrounding solvent (Fig. 3g). ${ }^{28,29}$ The morphology changes from graphite flake to the aggregated graphene nanosheets during LAL process was clearly demonstrated for off-GOQDs as shown in Fig. 3f-g. Off-GOQDs with a few nanometer size was formed after further ablation of LAL up to $30 \mathrm{~min}$ (Fig. $3 \mathrm{~h}$ ). HRTEM was performed to study structural characterization of the intermediates (i.e., graphene nanosheets) of on-GQDs and offGOQDs after 5 min ablation (Fig. S3 $†$ ). The graphene nanosheets derived from LFL process shows highly crystalline structure with well-defined lattice fringe of $0.24 \mathrm{~nm}$ and the average size of c.a. $400 \mathrm{~nm}$, while smaller average size of $30 \mathrm{~nm}$ with lower crystallinity was found for off-GQDs derived from LAL process. Much smaller size of the graphene intermediates for off-GQDs reveals that the formation of GQDs occurs via bottom-up process when high-power sonication is not used for PLA. These drastic differences in the morphology of the intermediate compounds clearly demonstrated that the formation of on-GQDs and off-GOQDs are mainly dominated by LFL and LAL processes, respectively. Interestingly, both of on- and offGOQDs exhibit similar morphology and size distribution after complete ablation process but clearly different chemical compositions (e.g. oxygen-rich functional groups for offGOQDs). This highlights that the formation of on-GQDs by LFL mechanism can effectively minimize surface oxidation of GQDs caused by thermal effects.

The optical properties, such as photoluminescence (PL), ultraviolet-visible (UV-vis) and time-resolved photoluminescence (TRPL) spectra, of on-GQDs and off-GOQDs are presented in Fig. 4. Fig. 4a shows the PL spectra of on-GQDs and 


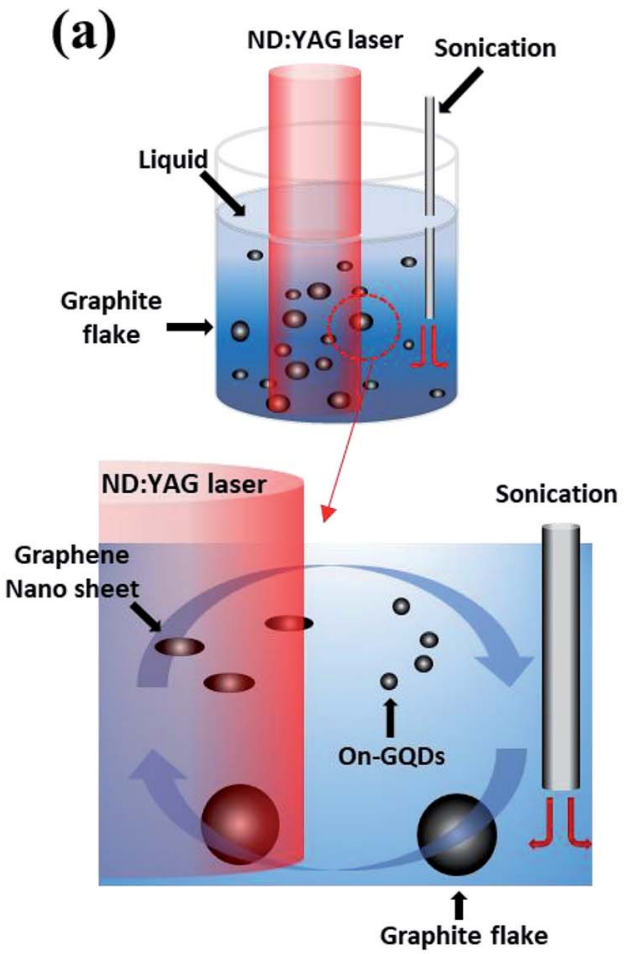

(c)

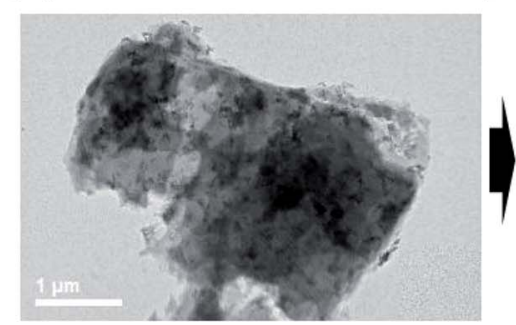

(f)

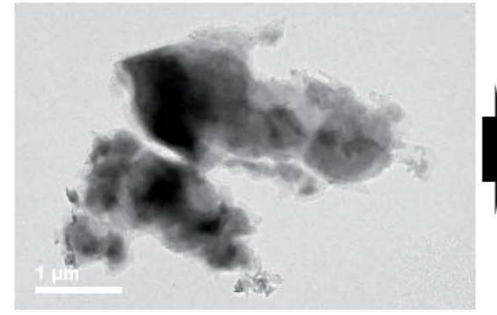

(g) (d)

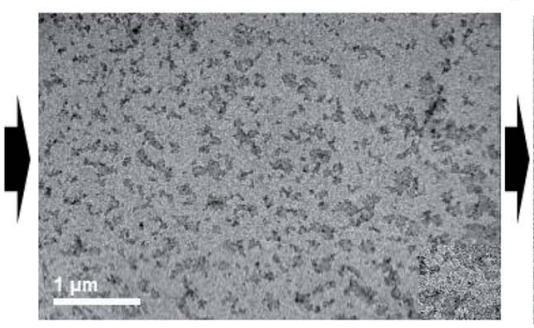

(e)

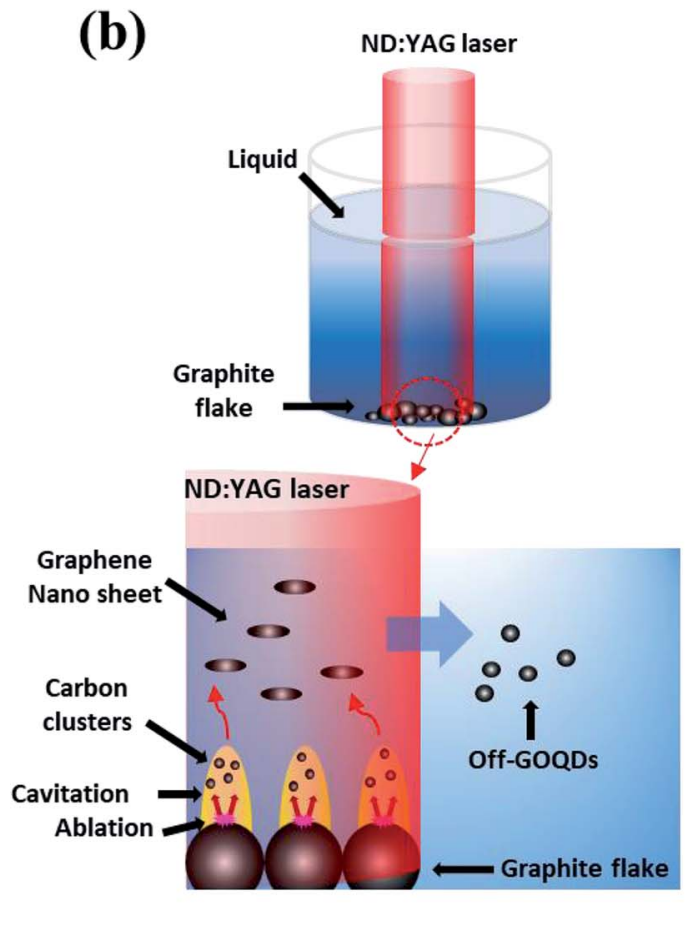

(h)
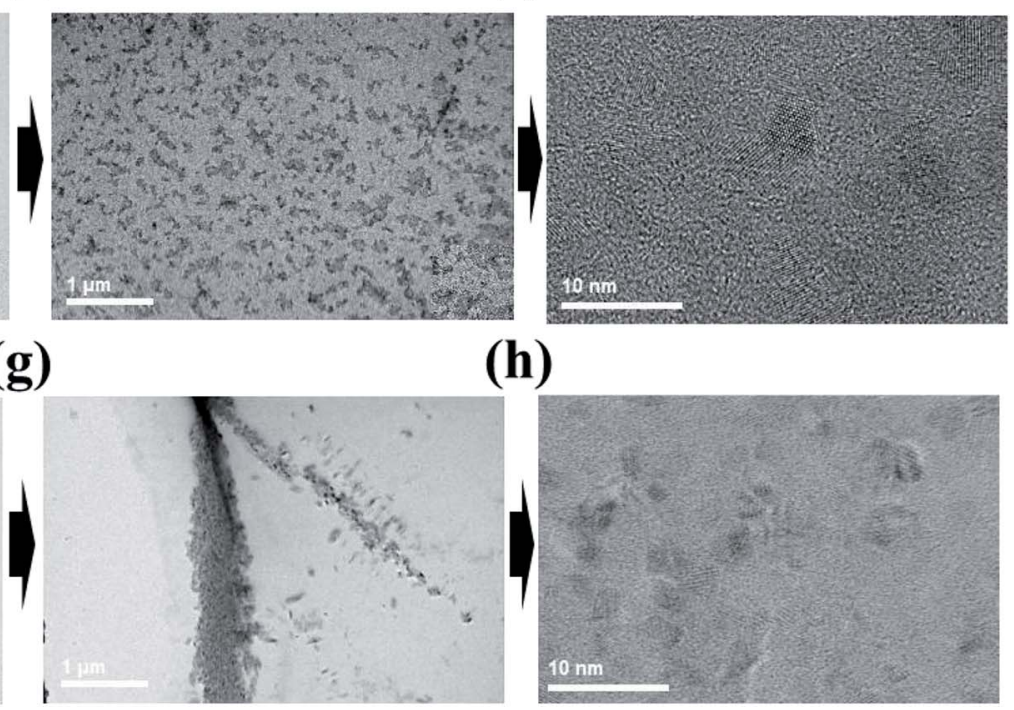

Fig. 3 Representative schematic for the possible mechanism of the (a) LFL and (b) LAL process. The time lamps ex situ HR-TEM images of graphite flake to on-GQDs and off-GOQDs conversions through the (c-e) LFL process and (f-h) LAL process, respectively.

off-GOQDs in ethanol. Compared to off-GOQDs, on-GQDs show stronger PL emission. The PL peak intensity of on-GQDs was about 2 times higher than that of off-GQDs at the same concentrations. The PL peak position of on-GQDs shows a shorter wavelength $(420 \mathrm{~nm})$ than that of off-GOQDs $(465 \mathrm{~nm})$ under the $360 \mathrm{~nm}$ excitation. The digital images of on-GQDs show clear blue emission (the inset in Fig. 4a, left digital image), while off-GOQDs exhibits a mixed emission of blue and green (the inset in Fig. 4a, right digital image). The PL of onGQDs and off-GOQDs is dependent on excitation wavelength
(Fig. S4a and $\mathrm{b}^{\dagger}$ ). PL peak of on-GQDs shifts to longer wavelength with the reduced peak intensity as excitation wavelength increased from 300 to $400 \mathrm{~nm}$, where a maximum peak was found at $420 \mathrm{~nm}$ with excitation wavelength of $340 \mathrm{~nm}$. Similarly, the emission peak of off-GOQDs shows an obvious red shift as excitation wavelength increases, while the PL intensity remains almost constant. In addition, PL-excitation (PLE) peaks were observed at around 260 and $360 \mathrm{~nm}$ for on-GQDs and offGOQDs, respectively. PLE peak of off-GOQDs is broader than that of on-GQDs while emission dependent PLE properties are 
(a)

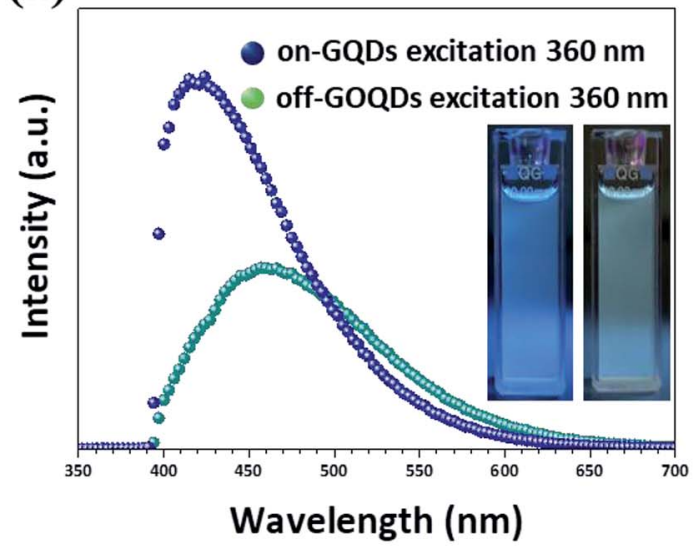

(c)

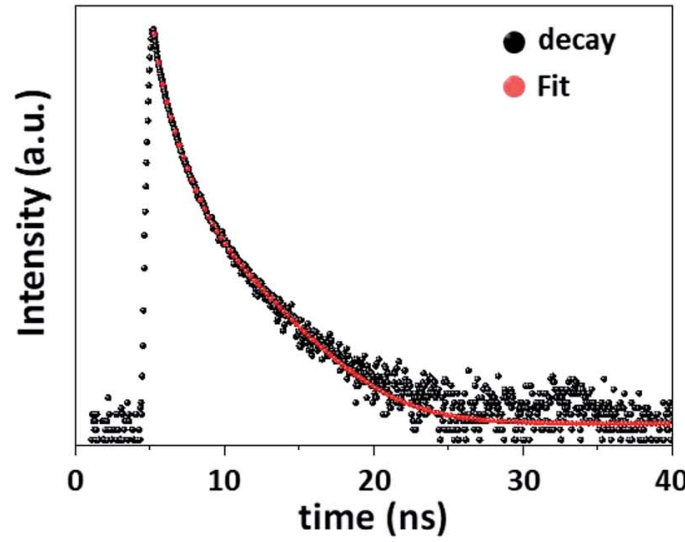

(b)

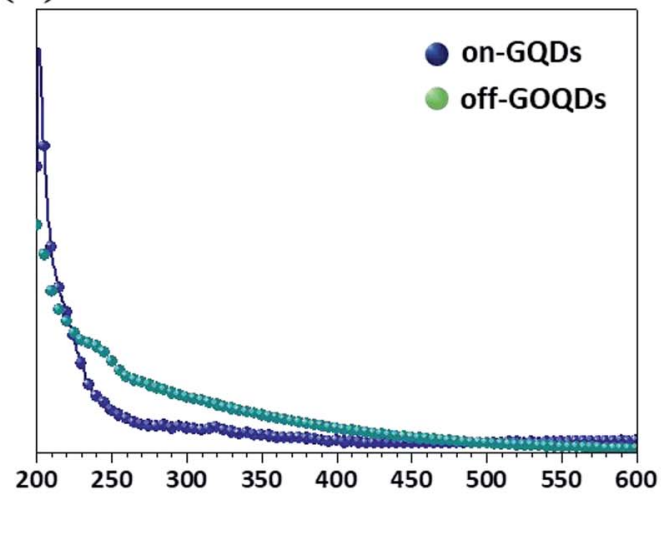

(d)

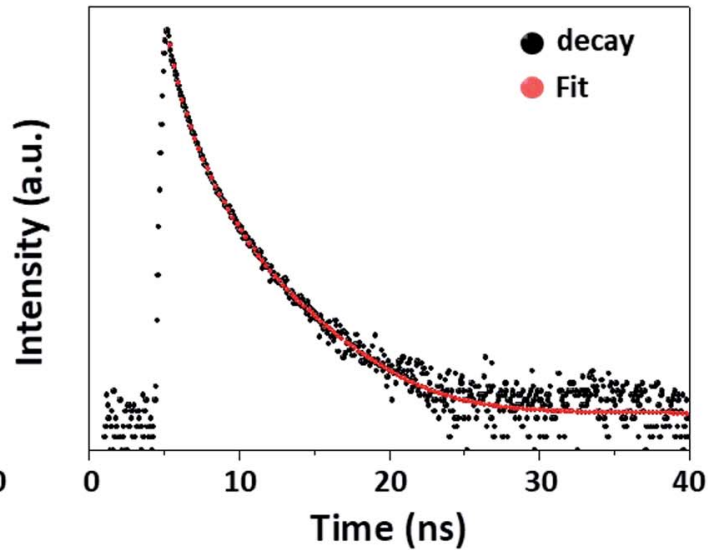

Fig. 4 Photoluminescence (PL) properties of (a) on-GQDs and off-GOQDs in ethanol. (b) UV-vis spectra of on-GQDs and off-GOQDs, respectively. TCSPC decay curves of the (c) on-GQDs and (d) off-GOQDs. The insets are digital images of on-GQDs and off-GOQDs under the excitation $360 \mathrm{~nm}$, respectively.

observed for the both samples (Fig. S4c and $\mathrm{d} \dagger$ ). Moreover, photo-stabilities of the on-GQDs and off-GOQDs were examined by continuous irradiation using UV lamp $(250 \mathrm{~W})$ with different time duration. As shown in Fig. $\mathrm{S} 4, \uparrow$ there were negligible changes in PL intensities both of on-GQDs (left) and off-GOQDs (right) when irradiation time increases up to $180 \mathrm{~min}$, demonstrating excellent optoelectronic properties of our GQDs.

UV-vis absorbance of off-GQDs shows a broad absorption spectrum with a gradual change up to $600 \mathrm{~nm}$ (Fig. 4b), which is similar to previously reported UV-vis absorbance results of graphene oxide QDs (GOQDs) with abundant oxygen-rich functional groups. ${ }^{30,31}$ Typically, UV-vis absorbance peak near at $220 \mathrm{~nm}$ is associated with $\pi \rightarrow \pi^{*}$ transition of $\mathrm{C}=\mathrm{C}$, while peaks at 240 and $340 \mathrm{~nm}$ are caused by $\mathrm{n} \rightarrow \pi^{*}$ transition of $\mathrm{C}=\mathrm{O}$ bond. Notably, only a weak shoulder at 240 and $340 \mathrm{~nm}$ is observed for on-GQDs, which is similar to UV-vis absorption spectra of pristine GQDs. ${ }^{32,33}$ These reveals that photoemissions of on-GQDs and off-GOQDs are largely affected by the oxygeous functional groups on the surface. In addition, the photo stabilities both of the on-GQDs and off-GOQDs were performed by under the UV lamp illumination from $250 \mathrm{~W}$ for different durations. A shown in Fig. S5a and $b, \dagger$ there were negligible changes in PL intensities both of the on-GQDs and off-GOQDs is observed.

To investigate the recombination mechanisms of on-GQDs and off-GOQDs, we carried out TRPL analysis (Fig. 4c and d). Table 1 depicts the values obtained by time-correlated single photon counting (TCSPC) characterization. Fluorescence decay curve is fitted with tri-exponential function (eqn (1)), where fluorescence decay occurs through three different relaxation pathways.

$$
\text { fit }=A+B_{1} \mathrm{e}^{\left(-t / \tau_{1}\right)}+B_{2} \mathrm{e}^{\left(-t / \tau_{2}\right)}+B_{3} \mathrm{e}^{\left(-t / \tau_{3}\right)}
$$

where $\tau$ is the fluorescence lifetime and $B$ represents amplitude of the corresponding lifetime. Chi-square $\left(X^{2}\right)$ value in the range

Table 1 Excitation emission values, $X^{2}$ value, excitation lifetimes, and their corresponding amplitudes for on-GQDs and off-GOQDs

\begin{tabular}{llllll}
\hline & $\begin{array}{l}\text { Ex/Emi } \\
(\mathrm{nm})\end{array}$ & Chisq & $\begin{array}{l}\tau_{1}(\mathrm{~ns}) / \\
B_{1}(\%)\end{array}$ & $\begin{array}{l}\tau_{2}(\mathrm{~ns}) / \\
B_{2}(\%)\end{array}$ & $\begin{array}{l}\tau_{3}(\mathrm{~ns}) / \\
B_{3}(\%)\end{array}$ \\
\hline On-GQDs & $370 / 450$ & 1.12 & $1.5 / 24$ & $4.6 / 54$ & $14 / 22$ \\
Off-GOQDs & $370 / 450$ & 1.18 & $1.2 / 16$ & $4.2 / 60$ & $15 / 24$
\end{tabular}


of 1.0-1.2 is generally assumed to be acceptable for fitting results. Typically, the emission that originates from defect states shows a longer recombination lifetime than that from intrinsic states. ${ }^{34}$ Also, among the three lifetimes, one is associated with an intrinsic state, while the other two are due to oxygen-rich functional groups on the surface of on-GQDs and off-GOQDs. Fluorescence lifetimes of on-GQDs and off-GOQDs are recorded at $450 \mathrm{~nm}$ with the excitation laser wavelength of $370 \mathrm{~nm}$. The lifetimes of on-GQDs are $\tau_{1}=1.5 \mathrm{~ns}(24 \%), \tau_{2}=4.6$ ns $(54 \%)$ and $\tau_{3}=14 \mathrm{~ns}(22 \%)$, whereas those of off-GOQDs are $\tau_{1}=1.3 \mathrm{~ns}(16 \%), \tau_{2}=4.2 \mathrm{~ns}(60 \%)$ and $15 \mathrm{~ns}(24 \%)$ (Table 1$)$. On-GQDs has a longer lifetime $\left(\tau_{1}\right)$ for the intrinsic state than off-GOQDs, and therefore the green emission can be effectively suppressed (Fig. 4a). Compared to on-GQDs, quantitative fraction of lifetimes for $\tau_{2}$ and $\tau_{3}$ was much higher for off-GOQDs probably due to the large amount of oxygen-rich functional groups on the surface of off-GOQDs, resulting in mixed green and blue emission. These results indicate that the produced onGQDs and off-GOQDs exhibit distinctly different optoelectronic properties due to the different formation mechanism by applying high-power sonication during the PLA process.

\section{Conclusions}

In conclusion, the proposed technique enables selectively producing GQDs and GOQDs by depending on the applying sonication during the PLA process, and they have a different formation mechanism. We found that homogeneous dispersion of graphite flakes due to high-power sonication resulted in uniform fragmentation of graphite by pulsed laser. On the other hands, when high-power sonication was not applied for PLA, graphite flakes were easily aggregated each other leading to the formation of plasma plumes at the surface of the graphite flake. According to compositional and structural analysis, chemical bonding of onGQDs is mainly composed of pure $\mathrm{sp}^{2}$ carbons with weak oxygen groups, while numerous oxygen-rich functional groups were presented on off-GOQDs. In addition, on-GQDs and off-GQDs showed clearly different optical properties. Off-GQDs exhibited blue to green mixed emission, while the emission of GQDs was distinct blue emission. We also proposed possible formation of on-GQDs and off-GQDs as laser fragmentation and laser ablation in liquid methods, respectively. We expect that the modified PLA method in this work will significantly reduce cost and time for the selective production of GQDs and GOQDs, which has a huge impact on designing various optoelectronic nanomaterials.

\section{Experimental}

\section{Materials}

Un retained nature graphite flakes were purchased by HQ graphene (Netherlands), and high purity ethanol (>99.9\%) was purchased from Sigma Aldrich.

\section{Preparation of GQDs and GOQDs}

On-GQDs and off-GOQDs were prepared by the simple and green PLA method using graphite in high-purity ethanol applying the tip-type sonication. In a typical process, $500 \mathrm{mg}$ of nature graphite flake (HQ graphene, Netherlands) was dispersed in $200 \mathrm{ml}$ of ethanol (>99\%, Sigma Aldrich) solutions. Tip-type sonication was subsequently performed during laser ablation for vigorous dispersion of graphite. The laser ablation was performed on the graphite suspension for $30 \mathrm{~min}$ by Qswitch ND:YAG laser system was employed at room temperature and in the air. The graphite suspension was ablated by a horizontal pulsed laser beam (355 nm, third harmonic) at a repetition rate of $10 \mathrm{~Hz}$. The pulsed width was $10 \mathrm{~nm}$, and the ablation energy of $1.5 \mathrm{~W}$. After the PLA treatment, the on-GQDs and off-GOQDs suspension was centrifuged at $15000 \mathrm{rpm}$ at 1 hours and were later filtered using syringe filters (Millipore, $0.22 \mu \mathrm{m}$ pore size). After that, the purified solutions were dried over night at $80^{\circ} \mathrm{C}$.

\section{Characterization of GQDs}

HR-TEM images were taken using a 2100F field emission gun TEM (JEM 2100F, JEOL, USA, $200 \mathrm{kV}$ ) for on-GQDs and offGOQDs samples. XPS spectra were recorded for both samples using VG ESCALAB 220i (Thermo scientific, USA). XPS survey and high-resolution scans were performed with the pass energies of $100 \mathrm{eV}$ and $20 \mathrm{eV}$, respectively. X-ray beam size was approximately $100 \mu \mathrm{m}$. On-GQDs and off-GOQDs samples for XPS measurement were prepared via a spin coating technique. Silicon ( $\mathrm{Si}$ ) substrate was used for spin coating. The rotation speed was adjusted to $3000 \mathrm{rpm}$. The samples were dried at room temperature for $2 \mathrm{~h}$ before the measurement. Room temperature PL spectra of on-GQDs and off-GOQDs were collected using a photoluminescence spectrophotometer (Horiba, Fluoromax-plus with $150 \mathrm{~W}$ xonon arc lamp) in the wavelength range from $300 \mathrm{~nm}$ to $700 \mathrm{~nm}$. Raman spectra were obtained for both samples using Raman spectrometer (LabRam Aramis, Horiba jobin Yvon, 514 nm Ar-ion laser used with a power of $1 \mathrm{~mW}$ ). A time-correlated single photon counting (TCSPC) spectrometer (Horiba Jobin Yvon, FluoroCube) was used to measure nanosecond lifetime.

\section{Conflicts of interest}

There are no conflicts do declare.

\section{Acknowledgements}

This work was partially supported by the Korea Institute of Industrial Technology as "The Project on Developing Technology of the multi-functional additive manufacturing and Supporting the SMEs of Applications" (KITECH JC-19-0003).

\section{Notes and references}

1 T. Gokus, R. R. Nair, A. Bonetti, M. Bohmler, A. Lombardo, K. S. Novoselov, A. K. Geim, A. C. Ferrari and A. Hartschuh, ACS Nano, 2009, 3, 3963-3968.

2 M. Zheng, S. Ruan, S. Liu, T. Sun, D. Qu, H. Zhao, Z. Xie, H. Gao, X. Jing and Z. Sun, ACS Nano, 2015, 9, 11455-11461. 
3 X. Yan, X. Cui and L. Li, J. Am. Chem. Soc., 2010, 132, 59445945.

4 A. Takami, H. Kurita and S. Koda, J. Phys. Chem., 1999, 103, 1226-1232.

5 A. Abbas, L. T. Mariana and A. N. Phan, Carbon, 2018, 140, 77-99.

6 V. Amendola and M. Meneghetti, Phys. Chem. Chem. Phys., 2009, 11, 3805-3821.

7 M. Bacon, S. J. Bradley and T. Nann, Part. Part. Syst. Charact., 2014, 31, 415-428.

8 K. A. Fernando, S. Sahu, Y. Liu, W. K. Lewis, E. A. Guliants, A. Jafariyan, P. Wang, C. E. Bunker and Y. P. Sun, ACS Appl. Mater. Interfaces, 2015, 7, 8363-8376.

9 Z. Gan, S. Xiong, X. Wu, T. Xu, X. Zhu, X. Gan, J. Guo, J. Shen, L. Sun and P. K. Chu, Adv. Opt. Mater., 2013, 1, 926-932.

10 Z. Gan, H. Xu and Y. Hao, Nanoscale, 2016, 8, 7794-7807.

11 Z. Huang, Y. Shen, Y. Li, W. Zheng, Y. Xue, C. Qin, B. Zhang, J. Hao and W. Feng, Nanoscale, 2014, 6, 13043-13052.

12 L. Li, G. Wu, G. Yang, J. Peng, J. Zhao and J. J. Zhu, Nanoscale, 2013, 5, 4015-4039.

13 Y. Li, Y. Zhao, H. Cheng, Y. Hu, G. Shi, L. Dai and L. Qu, J. Am. Chem. Soc., 2012, 134, 15-18.

14 S. Y. Lim, W. Shen and Z. Gao, Chem. Soc. Rev., 2015, 44, 362381.

15 L. Lin and S. Zhang, Chem. Commun., 2012, 48, 10177-10179.

16 T. N. Lin, K. H. Chih, C. T. Yuan, J. L. Shen, C. A. Lin and W. R. Liu, Nanoscale, 2015, 7, 2708-2715.

17 F. Liu, M. H. Jang, H. D. Ha, J. H. Kim, Y. H. Cho and T. S. Seo, Adv. Mater., 2013, 25, 3657-3662.

18 R. Liu, D. Wu, X. Feng and K. Mullen, J. Am. Chem. Soc., 2011, 133, 15221-15223.

19 T. Luo, P. Wang, Z. Qiu, S. Yang, H. Zeng and B. Cao, Chem. Commun., 2016, 52, 10147-10150.

20 D. Pan, J. Zhang, Z. Li and M. Wu, Adv. Mater., 2010, 22, 734738.
21 P. Russo, A. Hu, G. Compagnini, W. W. Duley and N. Y. Zhou, Nanoscale, 2014, 6, 2381-2389.

22 J. Shen, Y. Zhu, X. Yang and C. Li, Chem. Commun., 2012, 48, 3686-3699.

23 J. Shen, Y. Zhu, X. Yang, J. Zong, J. Zhang and C. Li, New J. Chem., 2012, 36, 97-101.

24 S. H. Song, M.-H. Jang, J. Chung, S. H. Jin, B. H. Kim, S.-H. Hur, S. Yoo, Y.-H. Cho and S. Jeon, Adv. Opt. Mater., 2014, 2, 1016-1023.

25 X. Tan, Y. Li, X. Li, S. Zhou, L. Fan and S. Yang, Chem. Commun., 2015, 51, 2544-2546.

26 V. Thongpool, P. Asanithi and P. Limsuwan, Procedia Eng., 2012, 32, 1054-1060.

27 D. Werner, S. Hashimoto and T. Uwada, Langmuir, 2010, 26, 9956-9963.

28 J. Xiao, P. Liu, C. X. Wang and G. W. Yang, Prog. Mater. Sci., 2017, 87, 140-220.

29 R. Ye, C. Xiang, J. Lin, Z. Peng, K. Huang, Z. Yan, N. P. Cook, E. L. Samuel, C. C. Hwang, G. Ruan, G. Ceriotti, A. R. Raji, A. A. Marti and J. M. Tour, Nat. Commun., 2013, 4, 2943.

30 H. Zeng, X.-W. Du, S. C. Singh, S. A. Kulinich, S. Yang, J. He and W. Cai, Adv. Funct. Mater., 2012, 22, 1333-1353.

31 D. Zhang, B. Gokce and S. Barcikowski, Chem. Rev., 2017, 117, 3990-4103.

32 H. Zhu, X. Wang, Y. Li, Z. Wang, F. Yang and X. Yang, Chem. Commun., 2009, 5118-5120, DOI: 10.1039/b907612c.

33 S. Zhu, J. Shao, Y. Song, X. Zhao, J. Du, L. Wang, H. Wang, K. Zhang, J. Zhang and B. Yang, Nanoscale, 2015, 7, 79277933.

34 S. Zhu, Y. Song, J. Wang, H. Wan, Y. Zhang, Y. Ning and B. Yang, Nano Today, 2017, 13, 10-14.

35 K. N. Kuding, B. Ozbas, H. C. Schniepp, R. K. Prud'homme, I. A. Aksay and R. Car, Nano Lett., 2008, 8, 36-41. 